\title{
Kinetics of interstitial defects in $\alpha$-Fe: the effect from uniaxial stress
}

\author{
Changwoo Kang ${ }^{\mathrm{a}}$, Qingyu Wang ${ }^{\mathrm{b}}$, Lin Shao ${ }^{\mathrm{a}, *}$
}

\begin{abstract}
Understanding defect kinetics in a stress field is important for multiscale modeling of materials degradation of nuclear materials. By means of molecular dynamics and molecular statics simulations, we calculate formation and migration energies of self-interstitial atoms (SIA) and SIA clusters (up to size of 5 interstitials) in alpha $\mathrm{Fe}$ and identify their stable configurations under uniaxial tensile strains. By applying uniaxial stress along [111], <111> oriented single SIA defects become more stable than <110> oriented SIA, which is opposite to stress-free condition. Diffusion of single SIA defects under [111] tensile stress is facilitated along [111] direction and the diffusion becomes one dimensional (1D). For SIA clusters, their diffusion under zero stress has gradual transition from three dimensional (3D) for small clusters to one dimensional (1D) for large clusters. Under the tensile stress along [111], the 3D to 1D transition is accelerated. For large SIA clusters, the stress effect is quickly saturated with less diffusivity enhancement in comparison with small SIA clusters.
\end{abstract}

Keywords: molecular dynamics simulations, strain, defect clustering, alpha Fe

$-1-$

(C) 2016. This manuscript version is made available under the Elsevier user license http://www.elsevier.com/open-access/userlicense/1.0/ 


\section{Introduction}

Numerous theoretical studies have been performed to obtain formation/migration energies of defects in alpha Fe by using molecular dynamics (MD) simulations, density function theory (DFT) and kinetic Monte Carlo simulations [1-11]. The defect kinetics are needed for multiple scale modeling of defect evolution in stainless steels for reactor applications. Majority of previous studies were limited to stressfree conditions. A few simulation studies considered the effect of stress on the defect generation and defect clustering in the early stage of displacement cascade formation and evolution [12-14]. A few limited atomic scale simulations were performed to compare the diffusion behaviors under stress $[4,15$, 16]. Ab-initio calculations were employed to investigate the stability and mobility of single SIA in alpha Fe under external deformation [17]. Previous studies also considered the changes of energy barrier for impurity diffusion under stress $[4,16,18]$.

For all previous studies on stress effects on defect formation and defect migration in alpha Fe, they were limited to single self-interstitial atom (SIA). The lack of knowledge on SIA clusters (containing more than one SIA) has been an issue for multi-scale modeling of defect development under conditions combining neutron damage and stress. One example is creep deformation of fuel cladding when strong fuel and fuel cladding interactions develop under high burnup. Understanding SIA and SIA clusters' behaviors are important to develop multiscale modeling approaches involving the coupling of microstructural changes and mechanical property changes. Small SIA clusters form in damage cascade stage and they are important precursor defects to form large stable defect clusters. In this study, we investigate the formation and migration energies of SIA defects from single SIA to 5-SIAs under uniaxial strain. The present study considers large strain up to $3 \%$, which is not uncommon for strain concentrated region at a cracking tip, which is one important degradation phenomenon for reactor in-core components. Stable defect configurations, defect formation/migration kinetics and diffusion mechanisms are discussed.

\section{Methodology}

We use LAMMPS [19] code for both molecular dynamics (MD) and molecular statistics (MS) simulations. MD simulations are used to simulate defect migration to extract diffusivity and diffusion energies as a function of temperatures. MS simulations are used to calculate defect formation energies of different defect configurations. The Fe interatomic potential used in the present study is an embedded atomic potential type and was developed by Mendelev et al. [20]. The potential was obtained by fitting to both ab initio total energy calculation results and experimentally measured properties. The supercell contains about $512000 \mathrm{Fe}$ atoms and periodic boundary condition is applied. In order to obtain migration 
energies, MD simulations are performed at temperatures from $350 \mathrm{~K}$ to $600 \mathrm{~K}$, which are high enough to obtain sufficient defect mobility but low enough to avoid dissociation of defect clusters. Under a given temperature the cell is first relaxed in NVT ensemble, then following by NPH ensemble. A stress is applied to the cell along [111] direction, to maintain a constant strain. After stress loading, a thermal relaxation is allowed in NVT ensemble. The cell stretching along [111] direction leads to shrinkage in both [1 $1 \overline{1} 0]$ and the $[11 \overline{2}]$ directions through a Poission's ratio of about 0.29 . The time step during simulation is fixed to $0.2 \mathrm{fs}$. After equilibration to a fixed temperature, a NVE microcanonical ensemble is used to simulate defect migration for a time period up to $40 \mathrm{~ns}$. The visual tool, OVITO [21], is used to record diffusion trajectories.

The migration energies of SIAs can be obtained by both MS and MD methods. For MS approach, the migration energies represent difference of total energy between the initial SIAs configuration and the SIAs at saddle points. Nudeged Elastic Band (NEB) method is used to study the kinetics of transition from the initial position to the final position [22-24]. The MD approach is more computational costly but it provides atomic-level details of SIAs' dynamics. The MD approach does not pre-select migration paths, and the migration energies are obtained from temperature dependence of diffusivities. Diffusivities are determined by

$D_{N}(T)=\frac{\overline{R^{2}}(T)}{2 n_{d} t}$

where $\overline{R^{2}}(T)$ is the mean-square displacement of the SIAs, $n_{d}$ is the dimensionality of the motion (1 to 3 ), and $t$ is the simulation time. The migration energies are extrapolated from Arrhenius expression,

$D_{N}(T)=D_{0, N} e^{-E_{m} / k_{B} T}$

where $k_{B}$ is Boltzmann's constant, $E_{m}$ is the migration energy, and $D_{0, N}$ is a pre-factor. The data analysis follows the independent interval method in order to alleviate issue from limited computational time [3, 8 , $9,25,26]$. In this approach, the total simulation time is divided into $\mathrm{K}$ short time segments. For each time interval of $\tau_{K}\left(=\frac{t}{K}\right)$, the square displacement $\overline{R_{l}^{2}}$ is calculated and the diffusion coefficient is obtained by considering the average of their square,

$D_{N}(T)=\frac{1}{K} \sum_{i=1}^{K} \frac{\overline{R_{l}^{2}}}{2 n \tau_{K}}(T)$

Care must be taken to appropriately select $\tau_{K}$, as discussed by Terentyev et al. [8]. The time interval $\tau_{K}$ must be long enough to include local correlations of defect motion (i.e. allowing diffusion in back and 
forth directions for 1-D diffusion), but not too long to significantly increase total computation time. Previous study show that obtained diffusivity decrease with increasing $\tau_{K}$, and become saturated for longer $\tau_{K}$. The shortest $\tau_{K}$ to reach such saturation should be used in order to minimize statistic error and maximize computational efficiency [8]. In the present study, we use $\tau_{K}$ of 3.3 to 400 ps depending on the defect sizes and temperatures.

\section{Result and discussion}

Many previous studies have been performed to understand diffusion mechanisms and obtain defect kinetics of SIAs and SIA clusters in $\alpha$-Fe and other metals [1, 2, 5, 6, 8-11, 27-33]. All these studies are limited to zero-strain conditions. Table I, II and III compare the structures of small SIAs, formation energies and migration energies from the present study with selected previous works (Table I and II for 0\% strain), although the major focus of the present work is for non-zero strain conditions.

Table I shows the structures of small SIAs identified from the MD simulations. The OVITO is used for visualization [21]. The structures of 1-5 SIAs under strain free condition are consistent with previous studies [1, 9, 31]. For 1-SIA and 2-SIA, only <110> is observed during migration. For 2-SIA, <110> sessile configuration is observed, which is featured with low diffusivity and high migration energy barrier at low temperatures (less than $500 \mathrm{~K}$ ). $\langle 110\rangle,\langle 111\rangle$, and sessile configurations are observed during the migration for 3-SIA and 4-SIA. The 4-SIA sessile configuration is relatively immobile. For 5-SIA, only $<111>$ is observed. Defect configurations may change at different temperatures and under different simulation times [9]. More detailed descriptions of SIA structures under strain free condition can be founded in previous studies $[1,2,9,31]$

The main differences between strain free and [111] strained conditions are alinement directions of defects. The 1 to 5 SIAs prefer to be aligned parallel to [111]. At $0 \%$ strain, the 1-SIA can take any <110> configuration. However, under [111] strain, only specific <110> configuration is preferred, which has small angle away from the [111] strain direction. Unlike strain free condition, 1-SIA under the strain

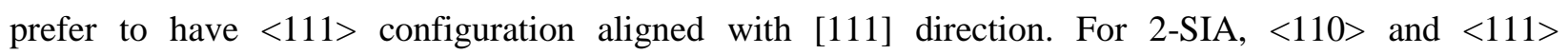
configurations are observed. However, under high strain, the sessile configurations are not observed. The

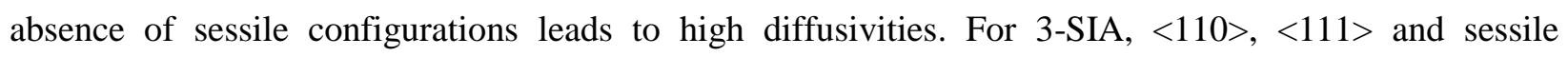
configuration are all observed. The sessile configuration is observed under $1 \%$ strain at $400 \mathrm{~K}$. The sessile configuration is highly immobile, similar to the 4-SIA sessile configuration at zero strain. For 4 and 5SIAs, only <111> configuration is observed under strain. As strain increases, small SIAs prefer to have $<111>$ rather than $<110>$ configurations. 
Table I. Observed configurations of 1-SIA to 5-SIA under strain free and strains along [111].

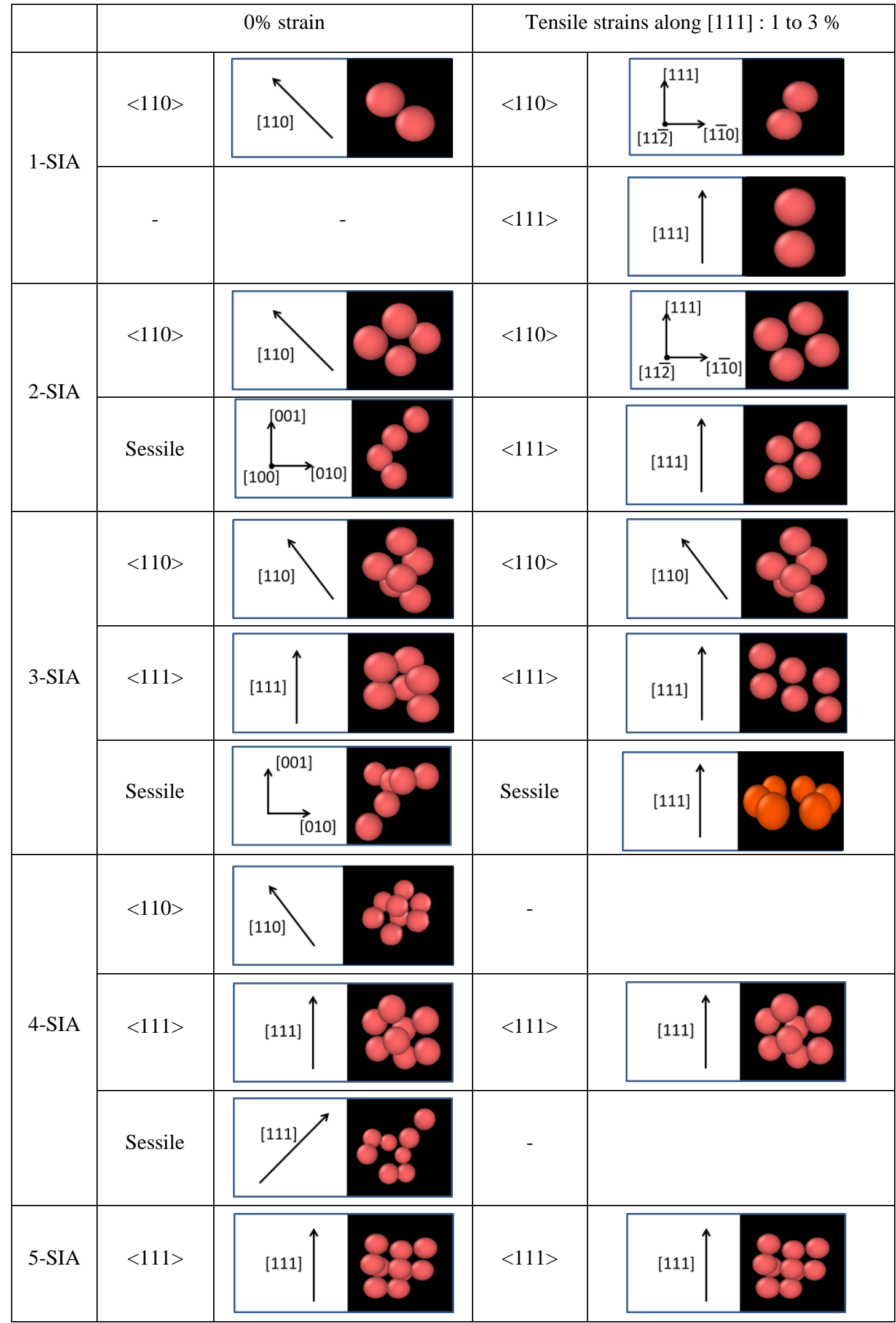


Table II. Formation energies in eV for 1-SIA to 5-SIA under 0\% strain.

\begin{tabular}{|c|c|c|c|c|c|c|c|}
\hline $\begin{array}{c}\text { Defect } \\
\text { size }\end{array}$ & Config. & $\begin{array}{c}\text { Present } \\
\text { work }\end{array}$ & $\begin{array}{c}\text { Anento et al. } \\
{[9]}\end{array}$ & $\begin{array}{c}\text { Terentyev } \\
\text { et al. [8] }\end{array}$ & $\begin{array}{c}\text { Marinica et } \\
\text { al. [31] }\end{array}$ & $\begin{array}{c}\text { Medelev et } \\
\text { al.[20] }\end{array}$ & $\begin{array}{c}\text { Willaime } \text { et } \\
\text { al. [32] }\end{array}$ \\
\hline \multirow{2}{*}{1} & $<110>$ & 3.52 & 3.57 & 3.52 & 3.52 & 3.52 & 3.75 \\
& $<111>$ & 4.02 & 3.97 & 4.01 & 3.94 & 4.02 & 4.45 \\
\hline \multirow{2}{*}{2} & $<110>$ & 6.19 & 6.28 & 6.23 & 6.21 & 6.21 & 6.68 \\
& $<111>$ & 6.73 & 6.86 & 6.76 & 6.74 & 6.74 & 7.43 \\
\hline \multirow{2}{*}{3} & $<110>$ & 8.85 & 9.00 & 8.87 & 8.84 & 8.84 & 9.49 \\
& $<111>$ & 9.37 & 9.39 & 9.36 & - & 9.37 & 10.01 \\
\hline \multirow{2}{*}{4} & $<110>$ & 10.92 & 11.04 & 11.05 & 10.94 & 11.05 & 11.66 \\
& $<111>$ & 11.07 & 11.18 & 11.22 & 11.14 & 11.36 & 11.77 \\
\hline \multirow{2}{*}{5} & $<110>$ & 13.24 & 14.12 & 13.42 & - & 13.25 & 14.18 \\
& $<111>$ & 13.20 & 13.47 & 13.49 & - & 13.36 & 13.88 \\
\hline
\end{tabular}

Table III. Migration energies in eV (diffusion prefactors in $10^{-8} \mathrm{~m}^{2} / \mathrm{s}$ ) for 1-SIA to 5-SIA under 0\% strain.

\begin{tabular}{|c|c|c|c|c|c|c|c|}
\hline $\begin{array}{c}\text { Defect } \\
\text { size }\end{array}$ & $\begin{array}{c}\text { Present } \\
\text { work }\end{array}$ & $\begin{array}{c}\text { Anento et al. } \\
\text { [9] }\end{array}$ & $\begin{array}{c}\text { Terentyev } \\
\text { et al. [8] }\end{array}$ & $\begin{array}{c}\text { Soneda et } \\
\text { al. [11] }\end{array}$ & $\begin{array}{c}\text { Marinica } \\
\text { et al. [31] }\end{array}$ & $\begin{array}{c}\text { J. Marian } \\
\text { et al. [4] }\end{array}$ & $\begin{array}{c}\text { Fu et al. } \\
\text { [6] }\end{array}$ \\
\hline 1 & $0.30(230)$ & $0.27(83.1)$ & $0.31(44.2)$ & $0.167(20.9)$ & 0.31 & $\begin{array}{c}0.127 \\
(19.4)\end{array}$ & 0.34 \\
\hline 2 & $0.41(1970)$ & $\begin{array}{c}0.36(351.6) \\
\text { for (T } \leq 500 \mathrm{~K}) \\
0.19(19.7) \\
\text { for (T > 500 K) }\end{array}$ & $0.33(243)$ & $0.083(7.32)$ & 0.33 & $\begin{array}{c}(14.8) \\
0.084\end{array}$ & 0.42 \\
\hline 3 & $0.13(11)$ & $0.14(12.1)$ & $0.14(3.35)$ & $0.065(5.57)$ & $0.33-0.38$ & $\begin{array}{c}0.074 \\
(14.2)\end{array}$ & 0.43 \\
\hline 4 & $0.14(17)$ & $0.15(12.3)$ & $0.16(3.11)$ & $0.100(7.93)$ & $0.05-0.14$ & $0.07 *$ & - \\
\hline 5 & $0.06(9)$ & $0.05(8.6)$ & $0.007(0.43)$ & $0.066(4.82)$ & - & $0.067^{*}$ & - \\
\hline
\end{tabular}

* The migration energy is deduced from their fitting equation. 
Table II and III show formation energies and migration energies of 1-SIA to 5-SIA, respectively. Formation energies of 1-SIA to 5-SIA are in good agreements. With increasing defect cluster sizes, formation energies are increasing. In consistent with these previous studies as shown in Table II, the present study shows that $\langle 110\rangle$ configurations are energetically more favorable than $\langle 111\rangle$ configurations. For SIAs of size from 1 to 3 , the energy difference between $\langle 111\rangle$ and $\langle 110\rangle$ is about 0.5 $\mathrm{eV}$. As for the migration energies (as shown in Table III), they are generally decreasing with increasing sizes. Migration energies obtained from Soneda et al. and Marian et al. have relatively large differences from others, due to different potentials used $[4,11]$. In generally, our results are in good agreements with previous studies $[8,9,31]$.

Figure 1(a-d) compare the defect formation energies as a function of uniaxial tensile strain along [111] for 1-SIA to 5-SIAs, respectively. Both $\langle 111\rangle$ and $\langle 110\rangle$ configurations are considered. All defect formation energies decrease with increasing strain, but the magnitudes of changes are different. $<111>$ configurations are more sensitive to strain than $\langle 110\rangle$. Hence, the energy gap between $\langle 111\rangle$ and $\langle 110\rangle$ configurations is reduced with increasing strain. As shown in Fig. 1a, the formation energies of <111> and <110> 1-SIA under zero strain are $4.02 \mathrm{eV}$ and $3.52 \mathrm{eV}$, respectively. At $3 \%$ strain, these two energies reduce to $3.19 \mathrm{eV}$ and $3.14 \mathrm{eV}$, respectively. For 2-SIA, $<110\rangle$ configuration is more favorable at zero strain. With increasing tensile strain, the energy gap between $\langle 110\rangle$ and $\langle 111\rangle$ configurations are reducing. At the highest strain of 3\%, as shown in Fig. 1b, the formation energy of $\langle 111\rangle$ 2-SIA is lower than $\langle 110\rangle$ 2-SIA. For 3-SIA, switching of favorable configurations from $\langle 110\rangle$ to $\langle 111\rangle$ occur at $2 \%$ strain, as shown in Fig. 1c. For 4-SIA, such change occurs at even smaller strain $<0.5 \%$ (Fig. 1d). For the largest defect 5-SIA, <110> and <111> configurations are comparable in formation energies for 0\% strain. But adding even small strain makes $<111>$ configuration more favorable (Fig. 1e). For small defect clusters (1-SIA to 3-SIA), the formation energies of both configurations show almost linear dependence on strain. For large defect cluster such as 5-SIA, formation energies of $\langle 110\rangle$ configuration deviate from Arrhenius temperature dependence and show an accelerated energy drop at higher strain $(>2 \%)$. It is worthy of the note that the trend of formation energy changes upon stress can be predicted by the elastic interaction between defects and applied stress fields. However, such estimation is difficult to consider the complexity caused by anisotropic strain relaxation associated with defect clusters. For multiscale modeling, it is necessary to have quantitative kinetics information as shown in Fig. 1. 

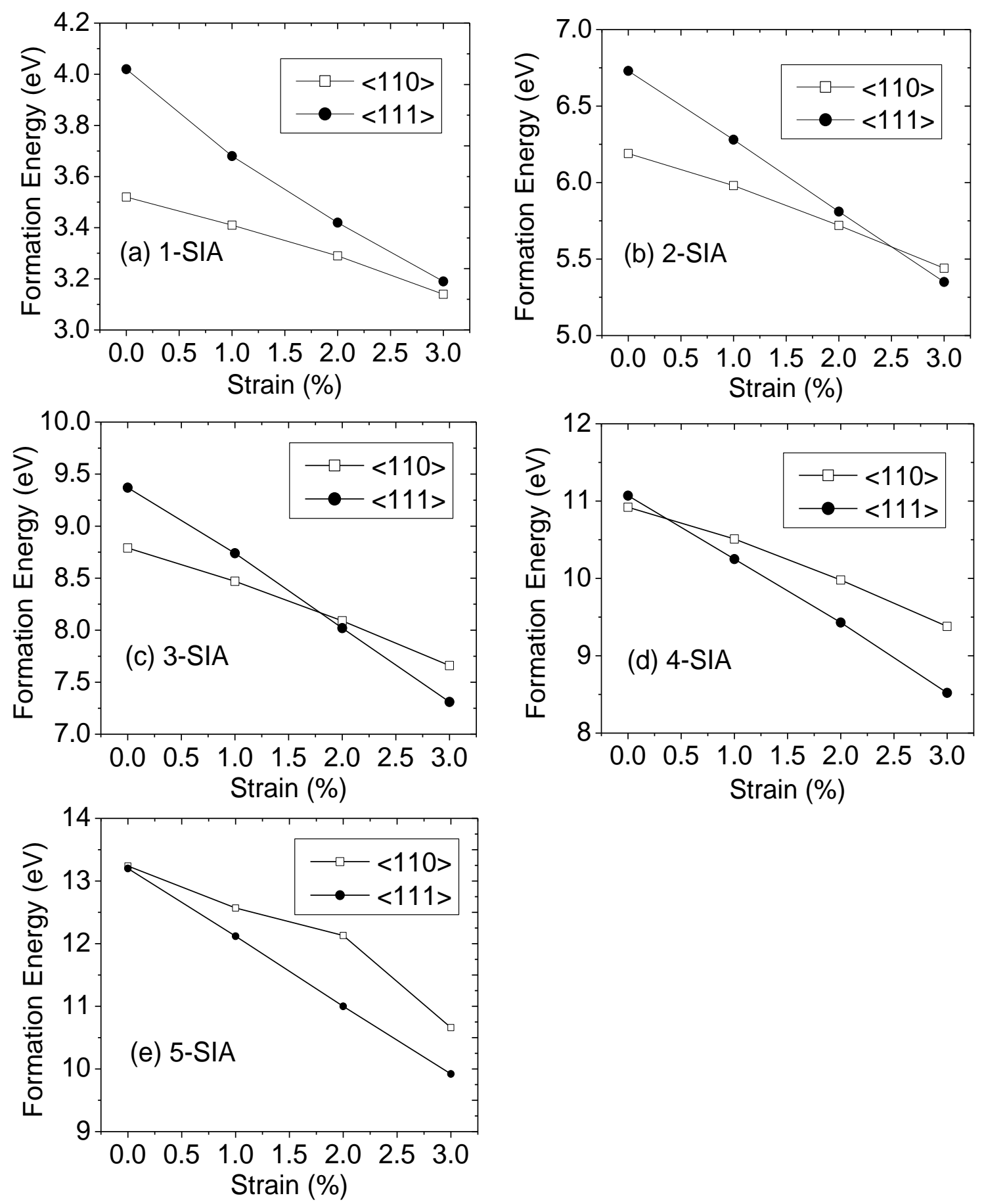

Fig. 1. Formation energies of $\langle 111\rangle$ and $\langle 110\rangle$ SIA defect clusters as a function of strain for cluster containing (a) single SIA, (b) 2 SIAs, (c) 3 SIAs, (d) 4 SIAs, and (e) 5 SIAs. 
(a) Mechanism A

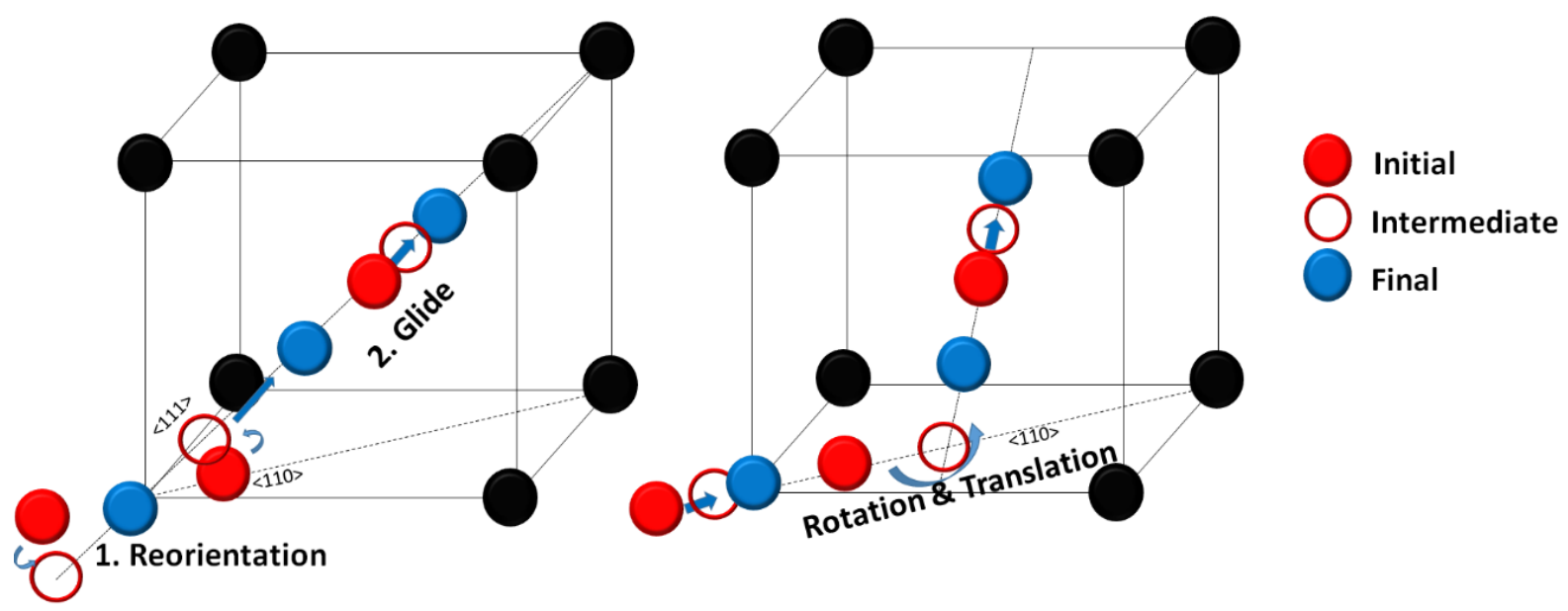

(b) Mechanism B

Fig. 2. Schematics of migration mechanisms for 1-SIA.

Under zero strain condition, there are two possible diffusion mechanisms for SIAs in $\alpha$-Fe. Figure 2 shows the migration mechanisms of A and B for 1-SIA. In the first mechanism (denoted as mechanism A), $<110>$ SIA(s) reorient to $<111>$ and then diffuse along $<111>$ direction. The migration energy is mainly contributed by the energy barrier required by reorientation $[1,8,10,11,29,34,35]$. In the second mechanism (denoted as mechanism B) which was first proposed by Johnson [36], <110> SIA(s) rotate rigidly and translate to other $<110>$ SIAs on a different plane. Recent DFT and MD studies suggested that mechanism $\mathrm{B}$ is more favorable $[5,8,9,31]$. One of interesting feature of the mechanism $\mathrm{A}$ is that 1-SIA can migrate with 1D resulted from the small formation energy difference between $\langle 111\rangle$ and $\langle 110\rangle$ configurations [8]. After one jump with the mechanism A, 1-SIA may translate consecutively without returning <110> configuration due to small energy barrier for $1 \mathrm{D}$ migration $[1,10,27,34]$. The mechanism $\mathrm{B}$, on the other hand, leads to $3 \mathrm{D}$ migration $[8,9,31]$.

We calculate the energy barriers for the diffusion mechanisms A and B, as shown in Fig. 3a and b, respectively. Energy barriers are calculated by using Molecular Statics (MS) and Nudged Elastic Band (NEB) methods [22-24]. Fig. 3a plots the energy barriers for reorientation of $\langle 110\rangle$ to $\langle 111\rangle$ configuration in the mechanism A. Previous DFT calculation showed such reorientation is unlikely due to relatively high energy barrier $(\sim 0.7 \mathrm{eV})[5,32]$. In consistency, the present study obtains an energy barrier of $0.50 \mathrm{eV}$ for $\langle 110\rangle$ to $<111>$ reorientation involved in the mechanism $\mathrm{A}$, and $0.31 \mathrm{eV}$ for $<110\rangle$ to $<110>$ migration involved in the mechanism B. In comparison, F. Willaime et al. obtained $0.50 \mathrm{eV}$ and $0.31 \mathrm{eV}$ by using MD simulations, and $0.76 \mathrm{eV}$ and $0.34 \mathrm{eV}$ by using DFT calculations [5, 32]; Terenvey et al. obtained $0.57 \mathrm{eV}$ and $0.34 \mathrm{eV}$ by using MD simulations for mechanisms A (reorientation only) and 
$\mathrm{B}$, respectively [8]. All these calculations show that mechanism B is energetically favored $(0.31 \mathrm{eV})$. As the strain along [111] increases, the energy barriers decrease for both mechanisms and the barrier difference between two mechanisms is reduced as well. At 3\% strain along [111], the two mechanisms become comparable $(0.214 \mathrm{eV}$ vs. $0.184 \mathrm{eV})$. The energy barrier, $0.184 \mathrm{eV}$, is higher than the MD result, $0.07 \mathrm{eV}$ (to be shown).

\section{(a) Mechanism A}

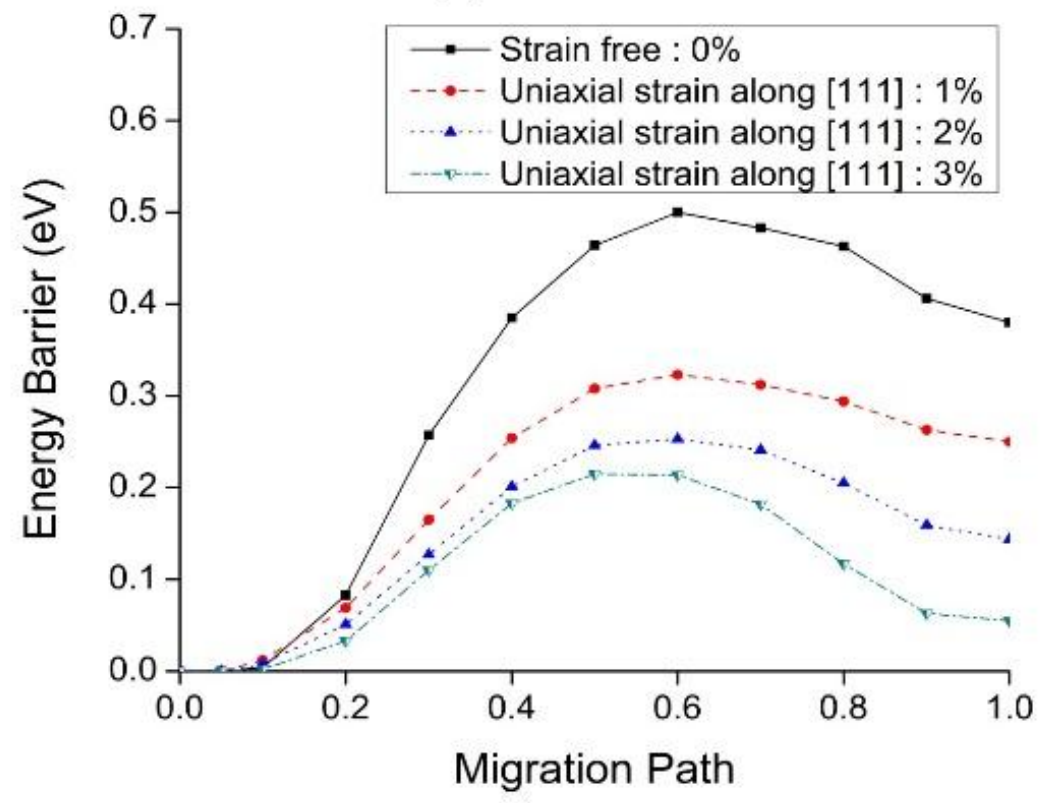

(b) Mechanism B

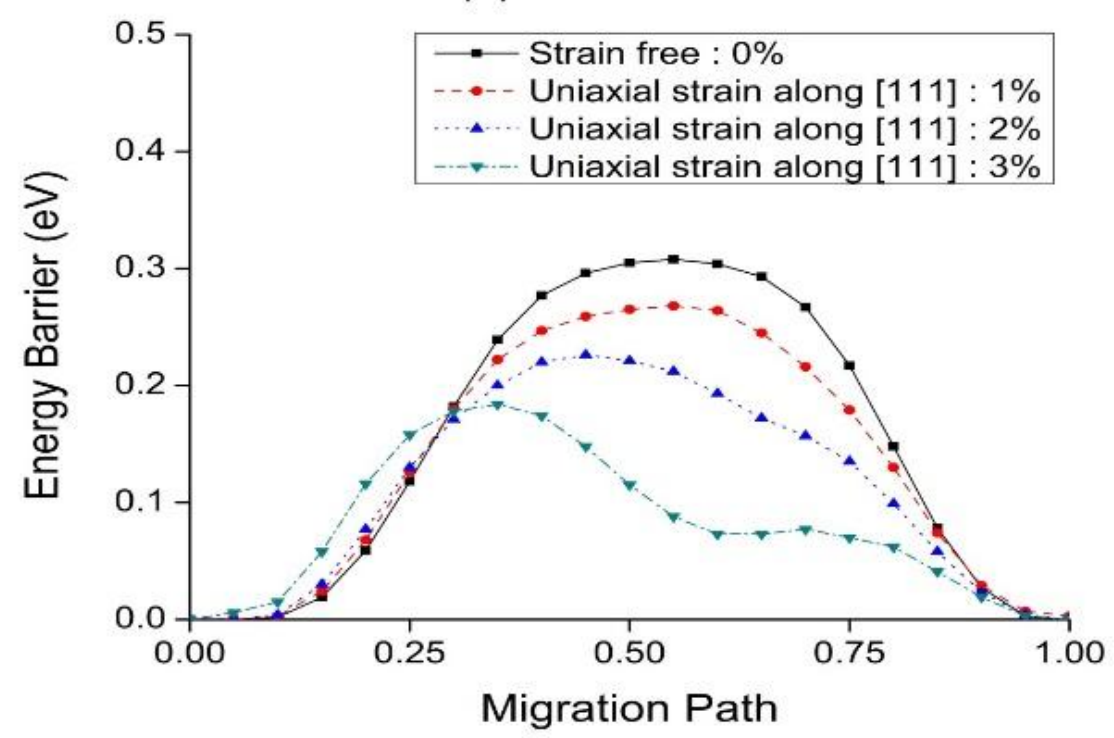

Fig. 3. The energy barriers of 1-SIA for (a) the migration mechanism A and (b) the migration mechanism. 

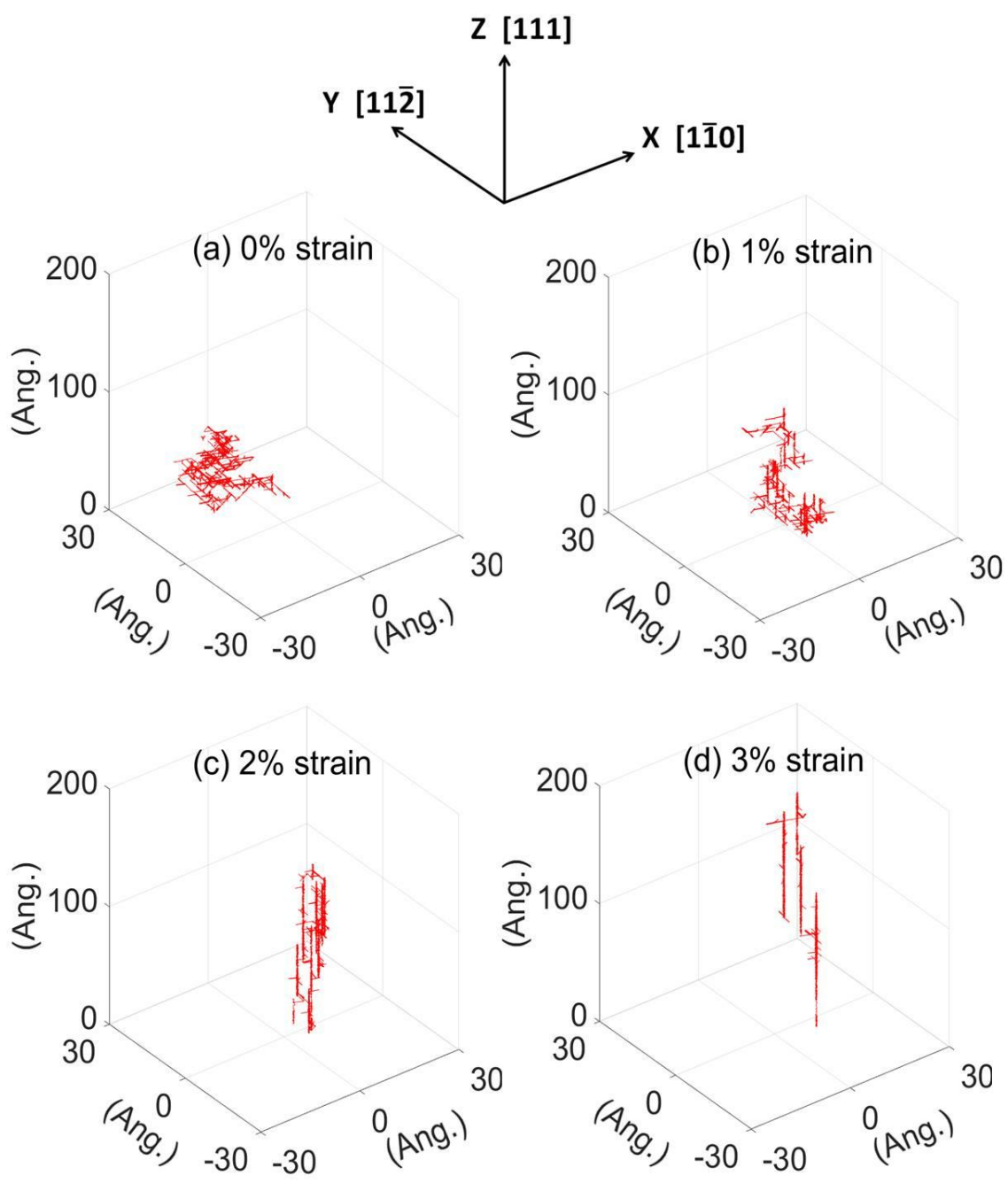

Fig. 4. 1-SIA migration trajectories under different uniaxial tensile strains along [111] direction at $500 \mathrm{~K}$.

Figures 4(a-d) compare single SIA migration trajectories under different tensile strains of $0 \%, 1 \%, 2 \%$ and 3\%, respectively. The $\mathrm{z}$ axis is [111] direction, $\mathrm{x}$ axis is [110] direction and $\mathrm{y}$ axis is [112] direction. The trajectories represent the movements of the center of mass of single SIA, used to calculate diffusion coefficient. As strain increases, the migration of 1-SIA gradually changes from 3D (Fig. 4 (a)) to 1D (Fig. 4 (d)). At $0 \%$ strain, the 1-SIA have only <110> configuration during the migration, which leads to 3D migration due to diffusion mechanism B. However, at 3\% strain, deformation induced asymmetric structural distortion favors certain alignments of 1-SIA. That is, the 1-SIA has different formation energy for different $\langle 110\rangle$ configurations. It causes $\langle 110\rangle$-SIA to choose specific $<110\rangle$ direction, which is aligned parallel to the strain direction [111]. Such alignment has been discussed before in simulation of 
cascade creation [12]. The 1D migration is due to the preference of parallel alignment of $\langle 110\rangle$ and $<111>1$-SIA to [111] strain direction. Although it is difficult to tell from Fig. 4, visual inspection at much smaller length scales show that for stain $>2 \%,<111>1$-SIA may occasionally change to $<110>1$-SIA. But $\langle 110\rangle$ 1-SIA quickly changes back to $\langle 111\rangle$ and re-start 1D diffusion. The time duration to stay as $<110\rangle$ configuration is much shorter at high strains. At highest strain of $3 \%$ the likelihood of $\langle 111\rangle$ to $<110>$ transition is further reduced. Diffusion is dominated by forward and backward movements along $<111>$ axis without returning back to $<110>$ configuration.

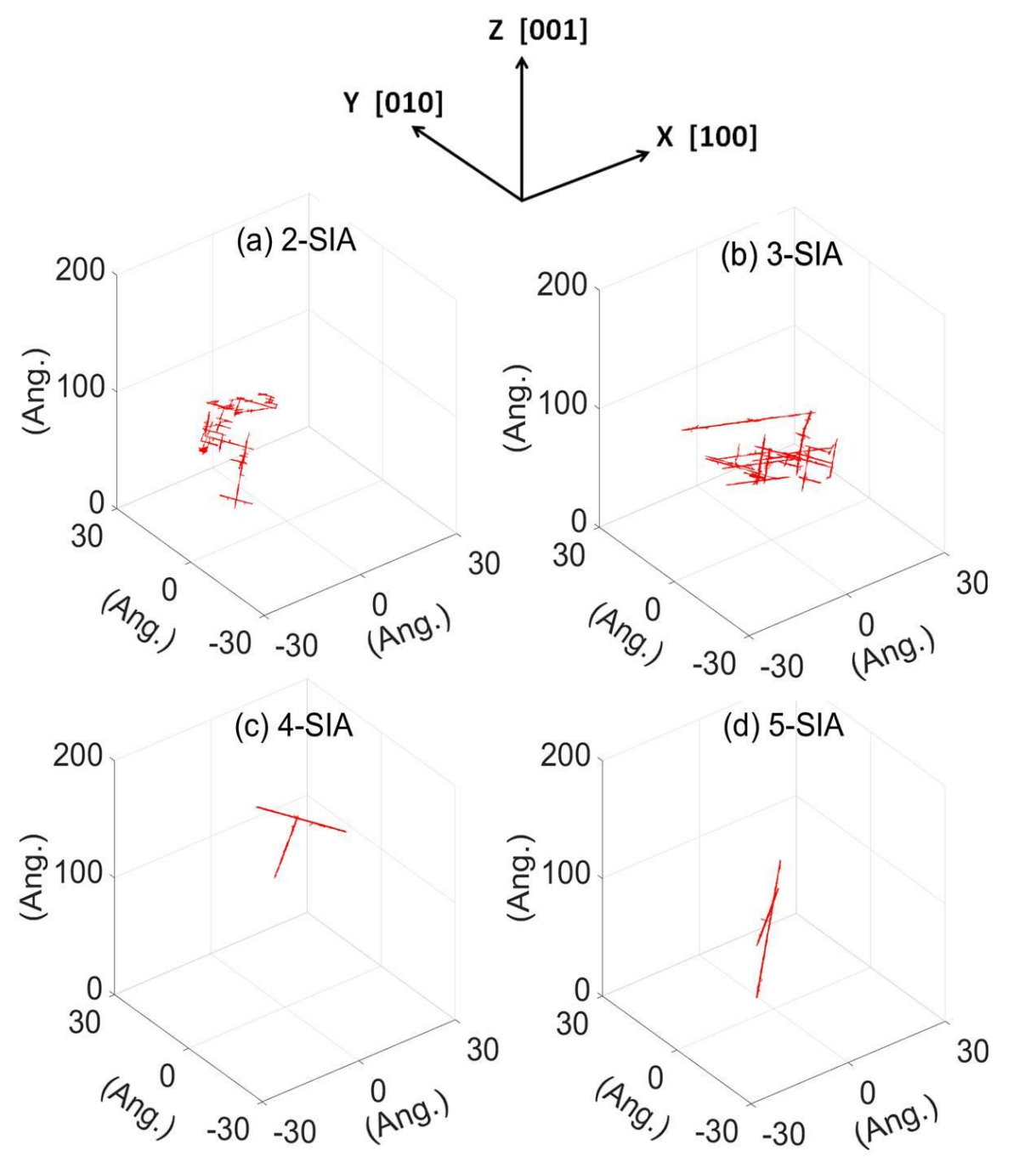

Fig. 5. Migration trajectories of (a) 2-SIA, (b) 3-SIA, (c) 4-SIA, and (d) 5-SIA under 0\% strain at $500 \mathrm{~K}$.

For defect clusters (from 2-SIA to 5-SIA), their diffusions under zero strain exhibit a transition from 3D (for small clusters) to 1D (for large clusters). Figure 5(a-d) plot the diffusion trajectories of 2-SIA to 5- 
SIA clusters. The $\mathrm{x}, \mathrm{y}, \mathrm{z}$ axes correspond to directions of [100], [010] and [001], respectively. All defect clusters show certain mixture of 3D and 1D diffusion. But their relative contributions to diffusion are sensitive to cluster sizes. For 2-SIA, as shown in Fig. 5a, diffusion favors 3D migration. For 3-SIA, diffusion is still a mixture of $3 \mathrm{D}$ and $1 \mathrm{D}$, but the contribution from $3 \mathrm{D}$ is decreasing, as evidenced by relatively longer migration distance (or equivalently, longer time duration) for 1-D migration along [111] axis, as shown in Fig. 5b. For larger clusters of 4-SIA and 5-SIA, as shown in Figs. 5c and 5d, 1D diffusion is dominant, featured by line-like diffusion trajectories. Defects may end its 1D migration by changing back to <110> configuration. But <110> SIAs immediately rotate into another <111> configuration and start another [111] 1D diffusion. These motion of small SIAs are consistent with previous works under strain free condition $[8,9]$.
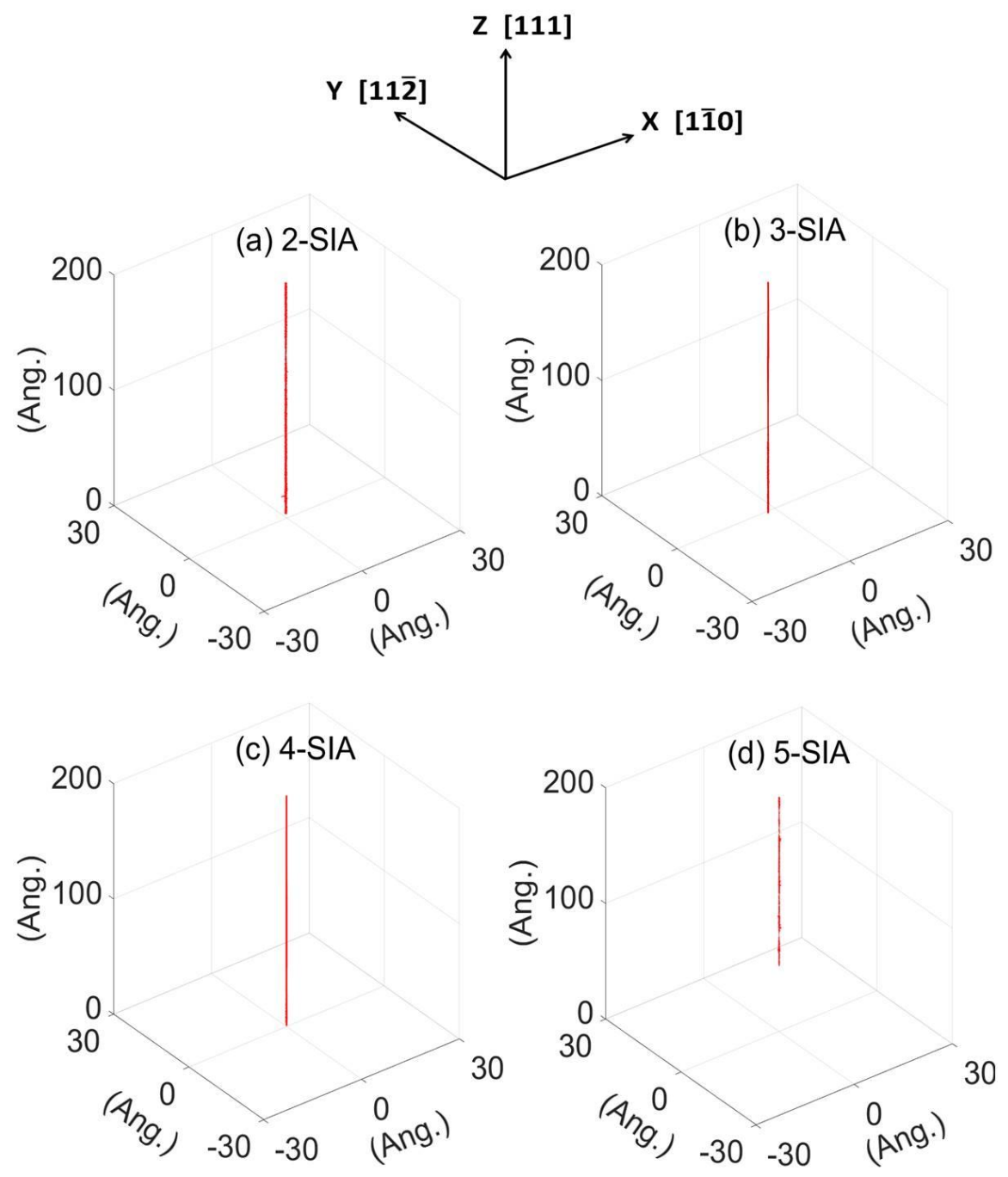

Fig. 6. Migration trajectories of (a) 2-SIA, (b) 3-SIA, (c) 4-SIA, and (d) 5-SIA under 3\% strain at $500 \mathrm{~K}$. 
Adding tensile strain promotes 1D migration, and the likelihood to end 1D migration through transition of $\langle 111\rangle$ to $\langle 110\rangle$ configurations is significantly reduced. Under high strains (3\%), no such configuration changes are observed. Figs. 6(a-d) plot the diffusion trajectories of 2-SIA to 5-SIA under 3\%

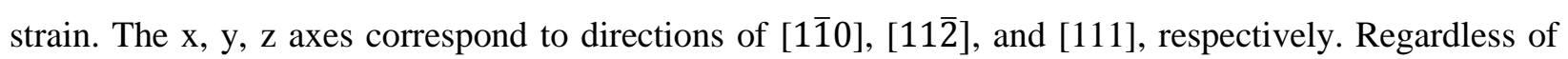
cluster sizes, all diffusion trajectories are featured by single straight-lines along [111] axis. The migration direction is matched to the strain direction and it shows fully 1D under 3\% strain along [111]. Again like 1-SIA, the formation energy differences between $\langle 111\rangle$ and $\langle 110\rangle$ of the small SIAs are reduced compared to that of unstrained crystals $(0 \%)$. Even, the $<111>$ for small SIAs have the lowest formation energy depending on strains as shown in Fig. 1 (b) to (e). Plus, under 3\% strains, all defects are aligned parallel to the strain direction [111]. Thus, the small SIAs show 1D fast diffusion to the applied strain direction. These migration behaviors under high [111] strain are similar to large SIAs (sizes more than 7) under zero strain. The fast 1D migration and its relative low migration energies for large SIAs have been observed in previous works $[1,4,8,9,11,28]$.

Figure 7(a-e) summarizes the diffusivities extracted from defect clusters of different sizes, from 1-SIA to 5-SIA, and under different strains, from $0 \%$ to $3 \%$. The effects of strain on motilities are significant, particularly for small clusters. As shown in Fig. 7a, for 1 SIA, diffusivities increase with increasing strain. At $350 \mathrm{~K}$ (corresponding to $1 / \mathrm{kT}=33.17 \mathrm{eV}^{-1}$ ), $3 \%$ strain enhances the diffusivity by more than two orders of magnitudes. The enhancements are less at higher temperatures. The temperature dependence shows an Arrhenius relation for a thermally activated process. The "apparent" activation energies are the largest for $0 \%$ strain, and are reduced with increasing strain, as reflected by slope changes of lines. We use "apparent" to emphasize that even diffusivities follow an Arrhenius expression governed by seemingly a single activation energy, the diffusion mechanisms behind can be a mixture of many different mechanisms. At $0 \%$ and $1 \%$ strain, 1-SIA defect diffuse dominantly through 3D mechanism B. At 2-3\% strain, it diffuses through a mixture of mechanism A and B as shown in Figure 3. These diffusion differences are supported by strain-induced formation energy changes (as shown in Fig. 1) and are the fundamental causes of diffusivity changes in both their magnitudes and activation energies.

For defect clusters larger than 1-SIA, the strain-induced diffusivity enhancements are relatively less. Introducing small amount of strain quickly enhances diffusivity to a level comparable to that under large strain. A higher strain results in a higher diffusivity, but the enhancement is less. Hence, the effect of strain is quickly saturated. Diffusivities at the highest strain of $3 \%$ are comparable among different cluster sizes. Fundamentally this is due to the fact that relative diffusion contributions of gliding with less migration energies are already significant for larger clusters under $0 \%$ strain (as evidenced by 3D to 1D diffusion transition shown in Fig. 3). Under strain, 1D diffusion is further promoted but its additional 
contribution to diffusivity enhancement is limited for large defect clusters. For the largest 5-SIA defect cluster, diffusivities under strain are very close to that without strain, since adding small amount of strain quickly lets $<111>$ configuration become energetically favorable and diffusion is exclusively governed by 1D migration.
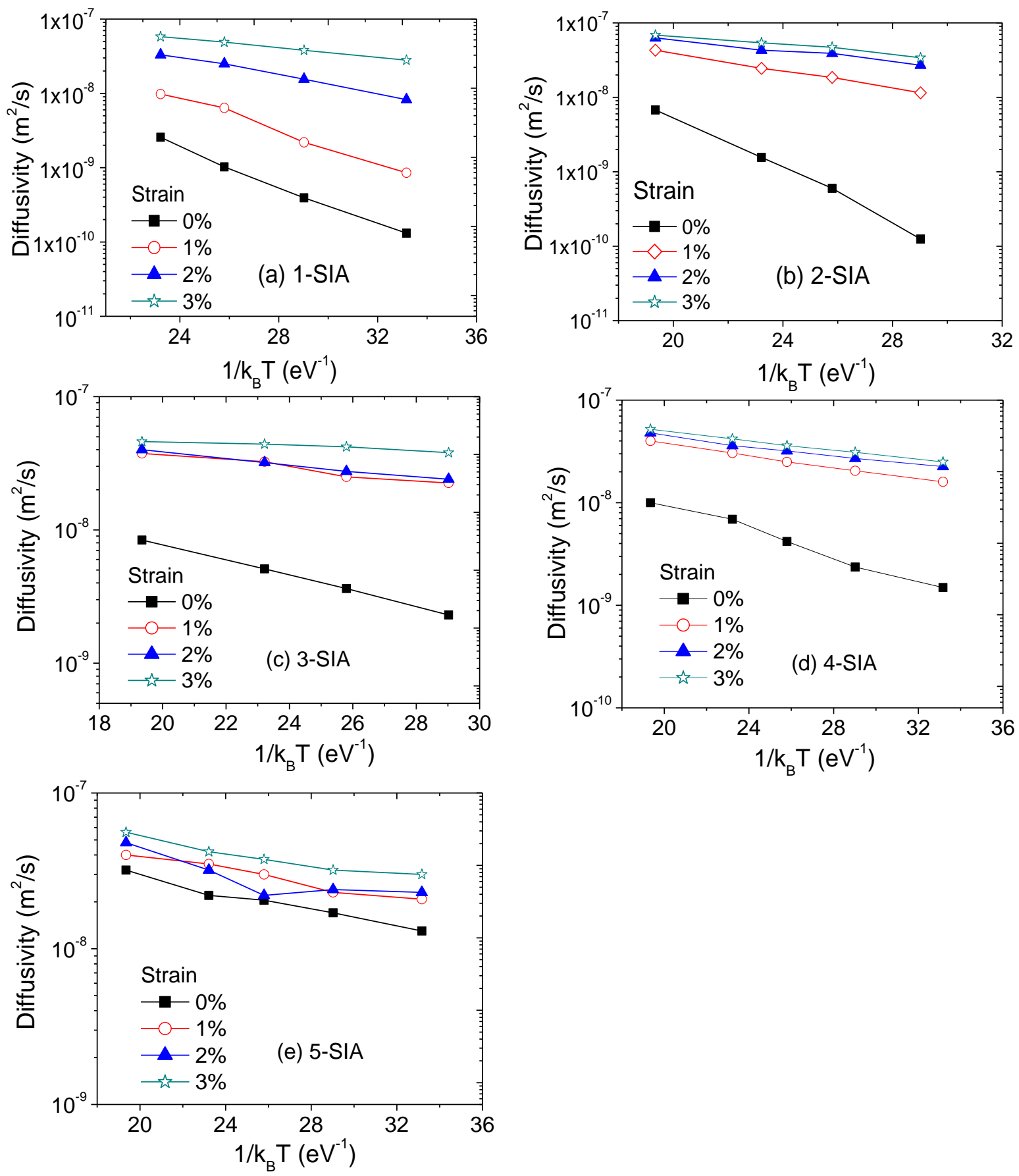

Fig. 7. Diffusion coefficients as a function of temperature under different tensile strain along $<111>$ direction for defect clusters containing (a) 1-SIA, (b) 2- SIAs, (c) 3-SIAs, (d) 4-SIAs, and (e) 5-SIAs. 
Table IV. Migration energies and diffusivity prefactors of SIAs defects.

\begin{tabular}{|c|c|c|c|c|c|c|c|c|}
\hline \multirow{2}{*}{$\begin{array}{c}\text { Defect } \\
\text { size }\end{array}$} & \multicolumn{6}{|c|}{$D_{0}\left(\times 10^{-8} \mathrm{~m}^{2} / \mathrm{s}\right)$ and $E_{m}(\mathrm{eV})$ at different tensile strains } \\
\cline { 2 - 9 } & \multicolumn{2}{|c|}{$0 \%$} & \multicolumn{2}{|c|}{$1 \%$} & \multicolumn{2}{c|}{$2 \%$} & \multicolumn{2}{c|}{$3 \%$} \\
\cline { 2 - 9 } & $D_{0}$ & $E_{m}$ & $D_{0}$ & $E_{m}$ & $D_{0}$ & $E_{m}$ & $D_{0}$ & $E_{m}$ \\
\hline 1 & 230 & $0.30 \pm 0.003$ & 383 & $0.23 \pm 0.005$ & 91 & $0.14 \pm 0.001$ & 33 & $0.07 \pm 0.001$ \\
\hline 2 & 1970 & $0.41 \pm 0.008$ & 58 & $0.14 \pm 0.003$ & 32 & $0.08 \pm 0.002$ & 28 & $0.07 \pm 0.001$ \\
\hline 3 & 11 & $0.13 \pm 0.003$ & 11 & $0.06 \pm 0.002$ & 11 & $0.05 \pm 0.001$ & 11 & $0.05 \pm 0.001$ \\
\hline 4 & 17 & $0.14 \pm 0.004$ & 14 & $0.07 \pm 0.001$ & 13 & $0.05 \pm 0.002$ & 12 & $0.05 \pm 0.003$ \\
\hline 5 & 9 & $0.06 \pm 0.002$ & 9 & $0.05 \pm 0.002$ & 11 & $0.05 \pm 0.002$ & 8 & $0.04 \pm 0.001$ \\
\hline
\end{tabular}

Table IV summarized the extracted migration energies and diffusivity prefactors of SIA defect clusters under different strains. Regardless of strain levels, migration energies of defect clusters in general become smaller with increasing cluster sizes. Under zero strain, diffusion energies change from $0.3 \mathrm{eV}$ for 1-SIA defect to $0.06 \mathrm{eV}$ for 5-SIA defect. Under 3\% tensile strain, the energies change from $0.07 \mathrm{eV}$ for 1-SIA defect to $0.04 \mathrm{eV}$ for 5-SIA defect. For the defects of the same size, increasing strain reduces migration energies. But the energy reduction is less for large defect clusters. For example, for 1-SIA defect, migration energy changes from $0.3 \mathrm{eV}$ under zero strain to $0.07 \mathrm{eV}$ under $3 \%$ strain. In a comparison, the changes are $0.06 \mathrm{eV}$ to $0.04 \mathrm{eV}$ for 5-SIA defect. For the diffusivity prefactor, it abruptly increases for 2SIA under strain-free $(0 \%)$ condition. Similar trend has been reported in previous studies [8,9]. The primary reason is the involvement of sessile configurations which are relatively immobile than other defect types.

It is worthy to point out that the present study has certain limits. Firstly, although the EAM potential used were able to reproduce qualitatively the DFT results, it may underestimate the formation energy difference between $\langle 110\rangle$ and $\langle 111\rangle$, which may impact on the diffusion properties predicted. Secondly, the present study is limited to strain effect along [111] only, since this direction is most effective to influence the stability difference between $<110>$ and $<111>$ SIA configurations [12]. Studies on diffusion behaviors under an arbitrary strain direction is beyond the scope of the present study. The effect of other strains could be very different.

The studies are important for multiscale modeling. Particularly for object kinetic Monte Carlo simulations which treat defect clusters as point-like defects. Both diffusivity enhancement and migration energy reduction for large defect clusters suggest that SIA clusters play an important role in defect 
clustering process, including agglomeration of small defects to larger ones, recombination of interstitialtype and vacancy-type defect clusters, and defect migration towards defect sinks such as grain boundaries. The dimensionality of SIAs' migration is important to defect clustering process [8]. This anisotropic diffusion causes big differences in microstructural evolution. Thus, the present study provides necessary fundamental understanding towards multiscale modeling.

\section{Conclusion}

We investigate the formation and migration energies of 1-SIA and SIA defect cluster under uniaxial [111] strain. Regardless of cluster sizes, both $\langle 111\rangle$ and $\langle 110\rangle$ oriented defects have reduced formation energies as a function of strain. Furthermore, <111> configuration is more sensitive to strain and its defect formation energies are reduced more significantly. For all defect cluster sizes, there are switching of stable configurations from $<110>$ SIA to $<111>$ SIA. The strain also reduces the energy barrier for $<110>$ SIA(s) to rotate into <111> SIA, which makes 1D migration along [111] more feasible. For 1-SIA, the migration dimension is $3 \mathrm{D}$ under zero strain, but change from $3 \mathrm{D}$ to $1 \mathrm{D}$ gradually with increasing strain. For SIA clusters, even under zero strain, their diffusion exhibit gradual transition from 3D to 1D at large sizes. By applying [111] tensile stress such transition starts at smaller defect sizes.

\section{Acknowledgements}

The research was supported by US Department of Energy's NEUP program, through grant no. DENE0008297. The modeling work is performed by using Texas A\&M High Performance Research Computing facility (http://hprc.tamu.edu/). 


\section{References}

[1] G.R.O. B.D. Wirth, D. Maroudas, G.E. Lucas, Energetics of formation and migration of self interstitials and self interstitial clusters in alpha iron, Journal of Nuclear Materials 244 (1997) 185-194. [2] Y.N. Osetsky, A. Serra, B.N. Singh, S.I. Golubov, Structure and properties of clusters of selfinterstitial atoms in fcc copper and bcc iron, Philosophical Magazine A 80(9) (2000) 2131-2157. [3] Y.N. Osetsky, Atomistic Study of Diffusional Mass Transport in Metals, Defect and Diffusion Forum 188-190 (2001) 71-92.

[4] J. Marian, B.D. Wirth, A. Caro, B. Sadigh, G.R. Odette, J.M. Perlado, T. Diaz de la Rubia, Dynamics of self-interstitial cluster migration in pure $\alpha-\mathrm{Fe}$ and Fe-Cu alloys, Physical Review B 65(14) (2002). [5] C.C. Fu, F. Willaime, P. Ordejon, Stability and mobility of mono- and di-interstitials in alpha-Fe, Physical review letters 92(17) (2004) 175503.

[6] C.-C. Fu, J.D. Torre, F. Willaime, J.-L. Bocquet, A. Barbu, Multiscale modelling of defect kinetics in irradiated iron, Nature Materials 4(1) (2004) 68-74.

[7] K. Kusunoki, Molecular-Dynamics Study of Self-Interstitial Diffusion in bcc-Iron, Materials Transactions 47(8) (2006) 1906-1909.

[8] D.A. Terentyev, L. Malerba, M. Hou, Dimensionality of interstitial cluster motion in bcc-Fe, Physical Review B 75(10) (2007).

[9] N. Anento, A. Serra, Y.N. Osetsky, Atomistic study of multimechanism diffusion by self-interstitial defects in $\alpha$-Fe, Modelling and Simulation in Materials Science and Engineering 18(2) (2010) 025008. [10] N. Soneda, T.D. de la Rubia, Defect production, annealing kinetics and damage evolution in $\alpha$-Fe: An atomic-scale computer simulation, Philosophical Magazine A 78(5) (1998) 995-1019.

[11] N. Soneda, T. Diaz de La Rubia, Migration kinetics of the self-interstitial atom and its clusters in bcc Fe, Philosophical Magazine A 81(2) (2001) 331-343.

[12] D.J.B. F. Gao, P.E..J. Flewitt, T.A. Lewis, The influence of strain on defect generation by displacement cascades in $\alpha$ iron, Nuclear Instruments and Methods in Physics Research B 180 (2001) 187-193.

[13] S. Di, Z. Yao, M.R. Daymond, F. Gao, Molecular dynamics simulations of irradiation cascades in alpha-zirconium under macroscopic strain, Nuclear Instruments and Methods in Physics Research Section B: Beam Interactions with Materials and Atoms 303 (2013) 95-99.

[14] B. Beeler, M. Asta, P. Hosemann, N. Grønbech-Jensen, Effects of applied strain on radiation damage generation in body-centered cubic iron, Journal of Nuclear Materials 459 (2015) 159-165.

[15] W.-L. Chan, R.S. Averback, Y. Ashkenazy, Anisotropic diffusion of point defects in metals under a biaxial stress field simulation and theory, Journal of Applied Physics 104(2) (2008) 023502. 
[16] D.S. Tchitchekova, J. Morthomas, F. Ribeiro, R. Ducher, M. Perez, A novel method for calculating the energy barriers for carbon diffusion in ferrite under heterogeneous stress, The Journal of chemical physics 141(3) (2014) 034118.

[17] Z. Chen, N. Kioussis, N. Ghoniem, D. Seif, Strain-field effects on the formation and migration energies of self interstitials in $\alpha$-Fefrom first principles, Physical Review B 81(9) (2010).

[18] X. Li, C. Gao, X.L. Xiong, Y. Bai, Y.J. Su, Hydrogen diffusion in $\alpha$-Fe under an applied 3-axis strain: A quantum manifestation, International Journal of Hydrogen Energy 40(32) (2015) 10340-10345.

[19] S. Plimpton, Fast Parallel Algorithms for Short-Range Molecular Dynamics, Journal of Computational Physics 117 (1995) 1-19.

[20] M.I. Mendelev, S. Han, D.J. Srolovitz, G.J. Ackland, D.Y. Sun, M. Asta, Development of new interatomic potentials appropriate for crystalline and liquid iron, Philosophical Magazine 83(35) (2003) 3977-3994.

[21] A. Stukowski, Visualization and analysis of atomistic simulation data with OVITO-the Open Visualization Tool, Modelling and Simulation in Materials Science and Engineering 18(1) (2010) 015012. [22] B.P.U. Graeme Henkelman, Hannes Jonsson, A climbing image nudged elastic band method for finding saddle points and minimum energy paths, Journal of chemical physics 113 (2000) 9901-9904. [23] H.J. Graeme Henkelman, Improved tangent estimate in the nudged elastic band method for finding minimyum energy paths and saddle points, Journal of chemical physics 113 (2000) 9978-9985.

[24] A. Nakano, A space-time-ensemble parallel nudged elastic band algorithm for molecular kinetics simulation, Computer Physics Communications 178(4) (2008) 280-289.

[25] M.W. Guinan, R.N. Stuart, R.J. Borg, Fully dynamic computer simulation of self-interstitial diffusion in tungsten, Physical Review B 15(2) (1977) 699-710.

[26] G.R.O. B.D. Wirth, D. Maroudas, G.E. Lucas, Dislocation loop structure, energy and mobility of self-interstitial atom clusters in bcc iron, Journal of Nuclear Materials 276 (2000) 33-40.

[27] M.V. Yu.N. Osetsky, A. Serra, S.I. Golubov, V. Priego, Computer simulation of vacancy and interstitial clusters in bcc and fcc metals, Journal of Nuclear Materials 251 (1997) 34-48.

[28] Y.N. Osetsky, D.J. Bacon, A. Serra, B.N. Singh, S.I. Golubov, One-dimensional atomic transport by clusters of self-interstitial atoms in iron and copper, Philosophical Magazine 83(1) (2003) 61-91.

[29] N. Soneda, S. Ishino, A. Takahashi, K. Dohi, Modeling the microstructural evolution in bcc-Fe during irradiation using kinetic Monte Carlo computer simulation, Journal of Nuclear Materials 323(2-3) (2003) 169-180.

[30] G.J. Ackland, M.I. Mendelev, D.J. Srolovitz, S. Han, A.V. Barashev, Development of an interatomic potential for phosphorus impurities in -iron, Journal of Physics: Condensed Matter 16(27) (2004) S2629S2642. 
[31] MArinica, (2011).

[32] F. Willaime, C.C. Fu, M.C. Marinica, J. Dalla Torre, Stability and mobility of self-interstitials and small interstitial clusters in $\alpha$-iron: ab initio and empirical potential calculations, Nuclear Instruments and Methods in Physics Research Section B: Beam Interactions with Materials and Atoms 228(1-4) (2005) 92-99.

[33] R.C. Pasianot, V.P. Ramunni, Small interstitials clusters migration in bcc metals: A Molybdenum model, Computational Materials Science 48(4) (2010) 783-789.

[34] R.E.S.G.R.O.B.D. Wirth, Primary damage formation in bcc iron, Journal of Nuclear Materials 251 (1997) 49-60.

[35] L. Malerba, Molecular dynamics simulation of displacement cascades in $\alpha$-Fe: A critical review, Journal of Nuclear Materials 351(1-3) (2006) 28-38.

[36] R.A. Johnson, Interstitials and Vacancies inaIron, Physical Review 134(5A) (1964) A1329-A1336. 